Titolo: "Verso nuove prospettive di ricerca e di cura: l'ipnosi come psicotecnica per migliorare e rinforzare le difese psicoimmunoendocrinologiche dell'organismo sottoposto a stress”.

Autore: Bossarelli A. ${ }^{1}$

Tipo: Breve rapporto

Keywords: ipnosi, difese immunitarie, stress;

Premessa

Questa è una breve revisione di alcuni articoli della letteratura scientifica internazionale sugli effetti dell'ipnosi in vari ambiti clinici, alcuni dei quali riguardano anche gli effetti positivi che l’ipnosi può indurre a proposito delle difese immunitarie e del sistema endocrino.

\title{
Studi considerati
}

Presso la University of Pennsylvania Medical School di Philadelphia è stata condotta una ricerca in cui 21 studenti di medicina del primo anno accademico sono stati avviati ad un percorso di formazione ipnotica con l'apprendimento dell'autoipnosi, finalizzato esclusivamente alla riduzione dello stress collegato allo studio e agli esami (Whitehouse et al., 1996). Osservando anche le reazioni immunitarie del campione, che non erano l'oggetto principale della ricerca, e confrontandole con un analogo campione di controllo che non aveva ricevuto nessuna suggestione, i ricercatori hanno rilevato che, nel periodo degli esami,

\footnotetext{
1 ASL di Piacenza - Dipartimento Salute Mentale e DP Infermiere Pedagogista e Psicologo Jr.

E-mail: a.bossarelli@email.it;
} 
si determinano delle modificazioni nella risposta immunitaria, che sono più favorevoli, in termini di numero e di attività delle cellule natural killer (NK) e di linfociti T, nel campione ipnotico sperimentale rispetto al campione di controllo. In particolare, all'interno del gruppo self-hypnosis, la qualità degli esercizi, per es. la profondità del rilassamento, è in grado di predire sia il numero delle cellule NK sia la loro attività.

Presso lo Imperial College of Science, Technology and Medicine di Londra, sono state condotte varie ricerche in tema riguardo l'impatto della suggestione sul funzionamento del sistema immunitario, riscontrando regolarmente degli effetti, limitati ma evidenti. Ad esempio: in una ricerca vengono somministrate a 16 soggetti, 10 sedute di ipnosi in cui viene suggestionato il rilassamento e l'attivazione delle capacità immunitarie, oltre ad un aumento generale dell'energia e della concentrazione. Si rileva quindi, confrontando il campione sperimentale con un gruppo di controllo nell'arco di 6 settimane, che lo stato di benessere soggettivo del campione è migliorato così come è cresciuta significativamente l'attività delle cellule NK (Gruzelier et al., 2001).

Un'altra ricerca ha studiato 25 pazienti che hanno contratto un tumore al seno (stadio 1 o 2), cui viene somministrato un programma di immaginazione guidata in stato ipnotico (Bakke, Purtzer e Newton, 2002). Durante la trance viene suggestionato un rilassamento progressivo, quindi vengono suggestionate alle pazienti delle metafore legate alla capacità di difendersi, come l'immagine di guerrieri che proteggono i confini o che sanno che c'è stata un'intrusione da parte di corpi estranei e che allora intervengono per distruggere gli invasori, ecc. Vengono misurati il numero e l'attività delle cellule NK prima e dopo la somministrazione del programma, riscontrando una significativa crescita dell’attività immunitaria.

Wood G.J. et al. (2003) hanno verificato l'ipotesi che l'ipnosi può modulare in modo differenziato sottoinsiemi di cellule $\mathrm{T}$, e che questo effetto è mediato dai cambiamenti ipotalamo-ipofisi-surrene (HPA) mediatori.

Sono state reclutate sette persone, sane, altamente suscettibili all'ipnosi, che hanno partecipato a 3 sessioni in un giorno e poi ripetute durante la seconda e terza settimana successiva: una di baseline e due con interventi ipnotici. Gli interventi sono stati somministrati in modo controllato (standardizzati) a vari livelli: nell'induzione, nelle suggestioni di rafforzamento dell'Io e per il funzionamento bilanciato e ottimale del sistema immuno-endocrinologico (SIE), infine per le suggestioni post-ipnotiche riguardo la gestione 
dello stress e il funzionamento del SIE. Ai soggetti sperimentali non è stata fornita nessun tipo di istruzione riguardo al funzionamento del sistema immunitario/endocrino, e nessuna informazione riguardo agli effetti attesi.

I campioni di sangue sono stati prelevati in cinque momenti dalle 8 alle 03 e sono stati analizzati per l'attivazione delle cellule T e citochine intracellulari (Interferone, interleuchina2, interleuchina-4) e mediatori dell'asse HPA (ACTH, cortisolo, e beta-endorfina). I linfociti $\mathrm{T}$ sono una componente centrale della risposta immunitaria adattativa e possiedono molte funzioni: riconoscimento dell'antigene, regolamento dell'immunità anticorpale e cellulomediata, soppressione di cellule bersaglio infette. Queste funzioni sono essenziali per il mantenimento dell'omeostasi immunologia.

Dopo l'intervento ipnotico si sono rilevati effetti immunologici statisticamente significativi: la percentuale di cellule T che esprimono IFN e IL-2 erano più bassi dopo l'ipnosi. Risposta di attivazione delle cellule T è stata positivamente correlata all' ACTH e alla b-endorfina, mentre l’ IFN è stato correlato positivamente con i livelli di cortisolo.

E' stato dimostrato anche che il cortisolo può raggiungere anche livelli molto bassi durante l'ipnosi ma solo per alcuni, ma non tutti, i soggetti altamente ipnotizzabili.

Differenze significative nei livelli di cortisolo sono stati anche trovati in soggetti altamente ipnotizzabili, quando fanno esperienze indotte, di stati emozionali intensi.

Anche se molto limitata, questa evidenza suggerisce che l'ipnosi ha alcune potenzialità nella modulazione dell'asse HPA. Comunque i risultati di questo studio preliminare, utilizzando un campione di piccole dimensioni, non hanno potuto dimostrare effetti statisticamente significativi dell' ipnosi sui tre mediatori HPA testati, cioè ACTH, cortisolo, o betaendorfina. I livelli di ACTH nella seconda e terza settimana sono stati inferiori rispetto al basale non solo alle 8:00 del mattino ma anche al 11:00 e 15:00 Questi cambiamenti suggeriscono che l'ipnosi può attenuare il rilascio di ACTH, molto probabilmente mediato attraverso una diminuzione di rilascio dell'ormone di rilascio della corticotropina (CRH).

Il presente studio potrebbe introdurre un modello per studiare in modo non farmacologico la modulazione della risposta delle cellule $\mathrm{T}$ e dei mediatori cellulari e molecolari coinvolti ed è di possibile interesse non solo per comprendere le interazioni psiconeuroimmunologiche coinvolte, ma anche per le potenziali applicazioni cliniche. Altri esperimenti sono in corso per affrontare alcune delle questioni sollevate da questo studio. 


\section{Conclusioni}

Negli studi citati finora sono stati esaminati solo alcuni degli effetti dell'ipnosi sul sistema endocrinologico e immunitario. Posso concludere osservando che ci sono prove evidenti che suggeriscono che gli interventi psicologici di questo tipo possono avere un effetto significativo sulle risposte fisiologiche e sui processi di malattia e che gli individui hanno la capacità di influenzare il loro sistema immunitario attraverso interventi come l'ipnosi autoipnosi. Tuttavia, non è ancora chiaro in quale specifico substrato fisiologico agisce l’ipnosi e quali sono i percorsi specifici degli effetti prodotti nel sistema immunitario. Altre ricerche quindi sono necessarie per approfondire questo settore di studio.

\section{Bibliografia}

Bakke, A.C., Purtzer, M.Z., Newton, P. (2002).The effect of hypnotic-guided imagery on psychological well-being and immune function in patients with prior breast cancer. Journal of Psychosomatic Research, 53(6),1131-7.

Gruzelier, J., Levy, J., Williams, J., Henderson, D. (2001). Self-hypnosis and exam stress: comparing immune and relaxation-related imagery for influences on immunity, health and mood. Contemporary Hypnosis 18, (2), 73-86.

Whitehouse, W. G., Dinges, D. F., Orne, E. C., Keller, S. E., Bates, B. L., Bauer, N. K., Morahan, P., Haupt, B. A., Carlin, M. M., Bloom, P. B., Zaugg, L., \& Orne, M. T. (1996). Psychosocial and immune effects of self-hypnosis training for stress management throughout the first semester of medical school. Psychosomatic Medicine, 58, 249-263.

Wood, G.J., Bughi S., Morrison J., Tanavoli, S., Tanavoli S., Zadeh, H.H. (2003) Hypnosis, differential expression of cytokines by t-cell subsets, and the hypothalamo-pituitary-adrenal axis. American Journal of Clinical Hypnosis, 45(3), 179-196. 